Polymer Journal, Vol. 8, No. 4, pp 377-384 (1976)

\title{
The Widths of a Polymer Chain
}

\author{
Keiji Moro and Nobuhiko SaItô \\ Department of Applied Physics, Waseda University, \\ Tokyo 160, Japan. \\ (Received February 23, 1976)
}

\begin{abstract}
By solving appropriate diffusion equation we calculate the width of a Gaussian polymer chain in one direction and the radius of the circumscribing sphere, which is defined as the sphere around the center of mass which includes the whole chain and yet makes contact somewhere on the chain. Various quantities defining the size of a Gaussian chain are compared.

KEY WORDS Gaussian Chain / Circumscribing Sphere / Diffusion Equation / Adsorption /
\end{abstract}

The statistical theory of polymer chains has made great progress over the past three decades. Theories have been developed, generally based on the detailed molecular structures of polymers, to construct partition functions of statistical mechanics. On the other hand, provided that the general statistical properties of polymer chains are of primary interest, we can adopt another approach, the method of the diffusion equation, which is more tractable in some instances. For example, the entanglement problems have been treated by Edwards ${ }^{1,2}$ and also by Saitô and $\mathrm{Chen}^{3}$ using the diffusion equation. Furthermore Edwards ${ }^{4,5}$ discussed the excluded volume effect and the collapse of a chain by the method of Wiener integrals and the selfconsistent field approximation, which led to a sort of diffusion equation. Saito, et $a l,,^{6} \mathrm{de}-$ veloped a statistical theory of stiff chains from a diffusion equation derived by a Wiener integral formulation.

The purpose of the present paper is to give other examples of this approach and to calculate the spans or widths to a polymer chain. Usually the size of a polymer chain is estimated by its average end-to-end distance or its radius of gyration around the center of mass. In addition to these conventional quantities, Hollingsworth ${ }^{7}$ defined an "average boundary" of a random coil chain, which is determined by the average distance between the first link and the link which is most distant from the first link. We here consider the average width of a random coil chain along one direction, defined by the average minimum distance between the two parallel boundary planes which enclose the entire chain in between (see Figure 1). This quantity is calculated by the diffusion equation method. We learned recently that almost the same approach to this problem had already been published by Weidmann, Kuhn, and Kuhn. ${ }^{8}$ Their results are special cases of our present calculations; furthermore, our method of summation of series using $\delta$ function (see Appendix A) is different from and more widely applicable in various problems than theirs. Then we calculate the average minimum radius of a sphere around the center of mass which contains the whole chain. Finally, various quantities specifying the size of a polymer chain are compared.

\section{WIDTH OF A STATISTICAL CHAIN ALONG ONE DIRECTION}

Let $W(\boldsymbol{r}, n) \mathrm{d} \boldsymbol{r}$ be the probability that the vector from one end to the other of a polymer chain of $n$ monomer units lies in the volume element $\mathrm{d} \boldsymbol{r}$ around the vector $\boldsymbol{r}=(x, y, z)$. If the end-to-end distance of a polymer chain obeys a Gaussian distribution, the distribution function $W(r, n)$ satisfies the following equation, provided that the degree of polymerization $n$ is regarded as a continuous variable:

$$
\frac{\partial W}{\partial n}=D V^{2} W
$$

where $D=b^{2} / 6$ and $b$ is the statistical bond length. This equation tells us that a polymer chain can be described in terms of a diffusion 


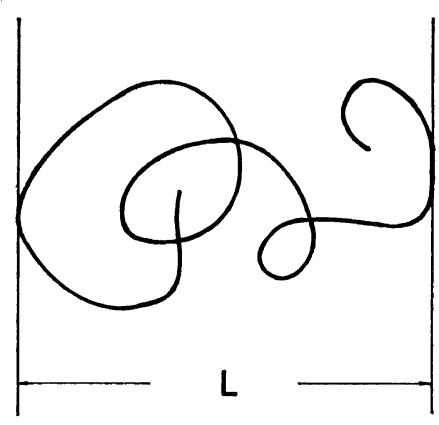

Figure 1. The width of a polymer chain along one direction.

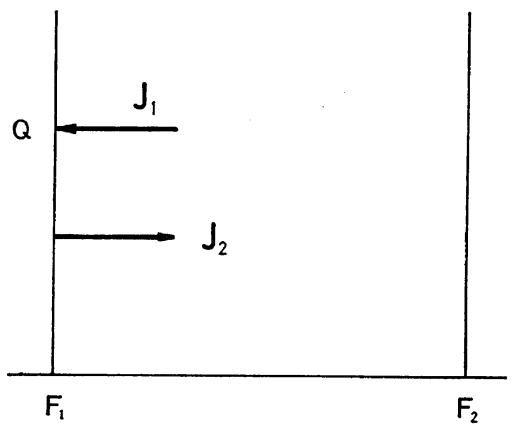

Figure 2. The boundary condition.

process when $n$ is regarded as time, as will be done below.

Now consider a pair of parallel planes $F_{1}$ and $F_{2}$ at a distance $L_{1}$ apart along one direction (say the $x$-direction), as shown in Figure 2 . The polymer chain, which reaches from the right at a point $Q$ on the plane $F_{1}$ in the terminology of the diffusion process, is assumed to go back to the right with probability $1-p$ or to be adsorbed on $F_{1}$ with adsorption coefficient $p$. Then the probability flow $J_{1}$ toward $F_{1}$ and the flow $J_{2}$ away from $F_{1}$ satisfy the following relations:

$$
\begin{gathered}
J_{2}=(1-p) J_{1} \quad\left(\text { on } \mathrm{F}_{1}\right), \\
J_{1}-J_{2}=D \frac{\partial W}{\partial x} \quad\left(\text { on } \mathrm{F}_{1}\right)
\end{gathered}
$$

On the other hand, $J_{2}$ is equal to the "average emission velocity" $\lambda$ multiplied by $W$ at $\mathrm{F}_{1}$,

$$
J_{2}=\lambda W \quad\left(\text { on } \mathrm{F}_{1}\right),
$$

where $\lambda$ is given by the average length of a step in the $x$-direction:

$$
\lambda=\int_{0}^{\pi / 2} b \cos \theta \sin \theta \mathrm{d} \theta=\frac{b}{2}
$$

Eliminating $J_{1}$ and $J_{2}$ from eq $2-4$ we have the boundary condition on $F_{1}$,

$$
\frac{\partial W}{\partial x}-a W=0, \quad a=\frac{p \lambda}{(1-p) D}
$$

similarly we have on $F_{2}$,

$$
\frac{\partial W}{\partial x}+a W=0
$$

For $p=1$, the chain is completely adsorbed on $\mathrm{F}_{1}$ and $\mathrm{F}_{2}$, and thus $W=0$. The chain terminates at the degree of polymerization for which it touches the boundary. On the other hand, for $p=0$, the chain is completely reflected at $F_{1}$ and $F_{2}$ and thus $\partial W / \partial x=0$ at the boundaries. The reasonable value of $p$ is $1 / 2$, because the boundary planes do not exist in real systems and thus the step of the chain always has equal probabilities for both + and - directions. The solution of eq 1 under the boundary conditions (5) and (6) is given by ${ }^{9}$

$$
W_{\mathrm{p}}\left(x, n, L_{1}\right)=\sum_{l=0}^{\infty} A_{l} \exp \left[-\frac{\alpha_{l}{ }^{2} D n}{L_{1}{ }^{2}}\right] z_{l}(x)
$$

where $\alpha_{l}$ 's are the solutions

$$
\frac{2}{\tan \alpha_{l}}=\frac{\alpha_{l}}{a L}-\frac{a L}{\alpha_{l}} \quad(a \neq 0)
$$

and

$$
\begin{aligned}
z_{l}(x) & =\frac{1}{c_{l}}\left\{\cos \frac{\alpha_{l}}{L} x+\frac{a L}{\alpha_{l}} \sin \frac{\alpha_{l}}{L} x\right\} \\
c_{l} & =\left[\frac{\alpha_{l}{ }^{2}+a^{2} L^{2}}{2 \alpha_{l}{ }^{2}} L+\frac{a L}{\alpha_{l}{ }^{2}} L\right]^{1 / 2}
\end{aligned}
$$

The arbitrary coefficients $A_{l}$ 's are determined by the initial condition. If we impose the condition that the starting point of the chain is distributed uniformly between the two planes, we put

Then we have

$$
W_{\mathrm{p}}\left(x, 0, L_{1}\right)=\frac{1}{L_{1}}
$$

$$
\begin{aligned}
& A_{l}=\frac{2 a L_{1}}{\alpha_{l}{ }^{2} c_{l}} \text { for odd } l \\
& =0 \text { for even } l
\end{aligned}
$$


The function $w_{\mathrm{p}}(n, L)$ defined by

$$
\begin{aligned}
w_{\mathrm{p}}(n, L) & =\int_{0}^{L} W_{\mathrm{p}}(x, n, L) \mathrm{d} x \\
& =4 \sum_{l=1, \text { odd }}^{\infty} \frac{1-\cos \alpha_{l}}{\alpha_{l}\left(\alpha_{l}+\sin \alpha_{l}\right)} \exp \left(-\frac{\alpha_{l}{ }^{2} D n}{L^{2}}\right)
\end{aligned}
$$

is the probability that the whole chain of $n$ monomer units is included between the two planes at the distance $L$ apart. Consequently, the probability density $f_{p}(L)$ that the width of a polymer chain is precisely $L$ is related to the function $w_{\mathrm{p}}(n, L)$ as follows:

$$
\frac{1}{L} \int_{0}^{L}(L-l) f_{p}(l) \mathrm{d} l=w_{p}(n, L)
$$

This yields the following equation:

$$
\begin{aligned}
f_{p}(L)= & L w_{\mathrm{p}}{ }^{\prime \prime}(L)+2 w_{\mathrm{p}}{ }^{\prime}(L) \\
= & 4 \sum_{l=1, \mathrm{odd}}^{\infty}\left[\frac { \alpha _ { l } { } ^ { 2 } \operatorname { s i n } ^ { 2 } \alpha _ { l } } { L ( \alpha _ { l } + \operatorname { s i n } \alpha _ { l } ) ^ { 5 } } \left\{\left(2 \cos \alpha_{l}+1\right) \alpha_{l}\right.\right. \\
& \left.\quad-\sin \alpha_{l}\left(\cos \alpha_{l}+2\right)\right\} \\
& +2 \frac{\alpha_{l}{ }^{3}\left(1-\cos \alpha_{l}\right)}{L^{3}\left(\alpha_{l}+\sin \alpha_{l}\right)^{4}}\left\{\left(2 \cos \alpha_{l}+1\right) \alpha_{l}\right. \\
& \left.\quad-\sin \alpha_{l}\left(\cos \alpha_{l}+2\right)\right\} D n \\
& \left.+4 \frac{\alpha_{l}{ }^{5}\left(1-\cos \alpha_{l}\right)}{L^{5}\left(\alpha_{l}+\sin \alpha_{l}\right)^{3}}(D n)^{2}\right] \exp \left[-\frac{\alpha_{l}{ }^{2}}{L^{2}} D n\right]
\end{aligned}
$$

By making use of this function $f_{p}(L)$, the first and the second moments of $L$ can be obtained, as shown in Appendix A. In particular, for $p=1$ and $p=1 / 2$,

$$
\begin{aligned}
\langle L\rangle= & 2 \sqrt{\frac{2}{3 \pi}} \sqrt{n} b \quad(p=1) \\
= & 2 \sqrt{\frac{2}{3 \pi}} \sqrt{n} b\left(1-\sqrt{\frac{\pi}{6}} \frac{1}{\sqrt{n}}\right. \\
& \left.+\frac{1}{3 n}-\frac{1}{9 n^{2}}+\cdots\right) \quad\left(p=\frac{1}{2}\right)
\end{aligned}
$$

and

$$
\begin{array}{rlr}
\left\langle L^{2}\right\rangle & =\frac{4}{3}(\ln 2) n b^{2}=0.9242 n b^{2} & (p=1) \\
& =0.8864 n b^{2} \quad(n=1000) \quad\left(p=\frac{1}{2}\right)
\end{array}
$$

One sees that for large $n,(17 b)$ tends to (17a). Weidmann, et al., discussed the case of $p=1$, and obtained eqs 17a and 18a. The case of $p=0 \quad(a=0)$ is of no interest in the present connection, since in this case we always have

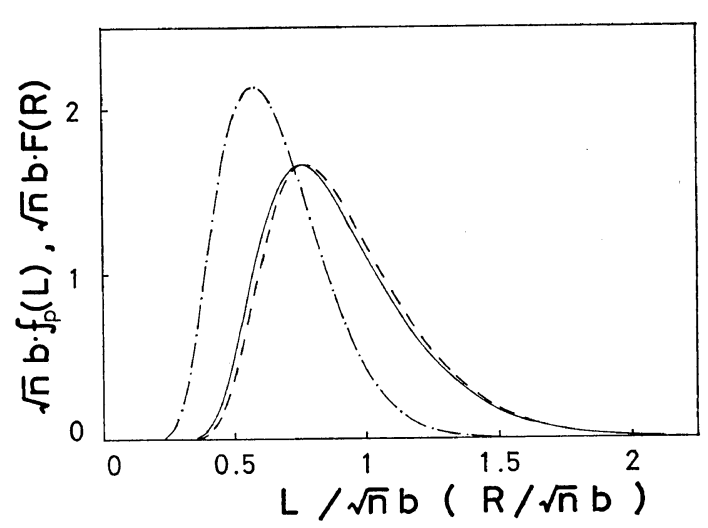

Figure 3. The probability densities, $\sqrt{n} b f_{1 / 2}(L)$ (solid line) and $\sqrt{n} b f_{1}(L)$ (dashed line), as a function of $L / \sqrt{n} b$. The probability density $\sqrt{n} b F(R)$ (dot-dashed line) as a function of $R / \sqrt{n} b$.

$W(x, n, L)=1 / L$, as is to be the case. The distribution functions $f_{p}(L)$ for $p=1 / 2$ and $p=1$ are shown in Figure 3. The difference is not large. From Figure 3, we obtain the value $L_{m}$ of $L$ for the maximum of $f_{p}(L)$. These values are, for $n=1000$,

$$
\begin{aligned}
& L_{m, 1}=0.78 \sqrt{n} b \text { for } p=1 \\
& L_{m, 1 / 2}=0.76 \sqrt{n} b \text { for } p=\frac{1}{2}
\end{aligned}
$$

Rubin ${ }^{10}$ considered the span of a lattice polymer. The average values of the span of the chain correspond to our eqs $17 \mathrm{a}$ and $18 \mathrm{a}$ in the limit $n \gg 1$, since he assumed completely absorbing walls at the boundaries. Later, Di Marzio and Rubin $^{11}$ employed parameters $a=\left(s-s_{0}\right) / s$ and $e^{\theta}\left(\theta=\varepsilon / R_{\mathrm{B}} T\right)$ in their adsorption problem of a polymer chain on cubic lattices (such as bcc, fcc, and sc lattices) between two layers, where $s$ and $s_{0}$ are the coordination number and the number of nearest neighbors of the lattice, respectively, and $\varepsilon$ is the adsorption energy of the surface layers. Their parameters $a$ and $e^{\theta}$ are related to our $p$ and $\lambda$, as

$$
\begin{gathered}
(1-a) e^{\theta}+\frac{1}{2} a e^{\theta}=(1-p) \\
\frac{1}{2} a=\lambda / b
\end{gathered}
$$

One of the simplest cases, $a=1$ (bcc lattice) and $\theta=0$ (energetically neutral boundaries), corresponds to our case $p=1 / 2$. 


\section{RADIUS OF THE CIRCUMSCRIBING SPHERE OF A POLYMER CHAIN}

Now consider the minimum radius of the sphere around the center of mass which contains the whole chain. Let $s_{j}$ be the vector from the center of mass to the $j$ th monomer (see Figure 4). In the Gaussian chain of $n$ monomers, the distribution function of the vector $s_{j}$ is also a Gaussian function: ${ }^{12-14}$

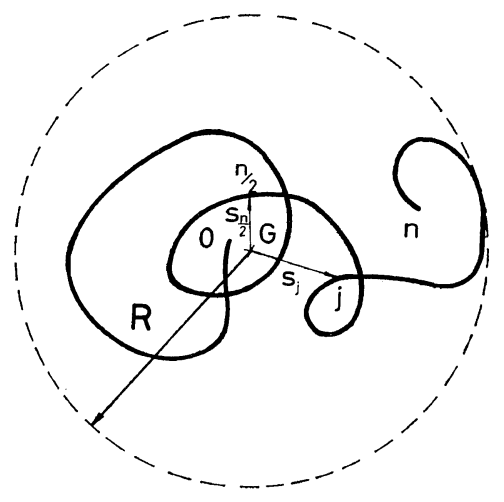

Figure 4. The circumscribing sphere of a polymer chain around the center of mass. The vector $s_{j}$ is the vector from the center of mass to the $j$ th segment.

$$
W\left(\boldsymbol{s}_{j}, n\right)=\left(\frac{3}{2 \pi\left\langle s_{j}{ }^{2}\right\rangle}\right)^{3 / 2} \exp \left[-\frac{3{s_{j}}^{2}}{2\left\langle s_{j}{ }^{2}\right\rangle}\right]
$$

with the second moment,

$$
\left\langle s_{j}{ }^{2}\right\rangle=\frac{b^{2}}{n}\left\{\left(j-\frac{n}{2}\right)^{2}+\frac{n^{2}}{12}\right\}
$$

If we choose $j=n / 2$ and put $\boldsymbol{r}=\boldsymbol{s}_{n / 2}(r=|\boldsymbol{r}|)$, we have,

$$
\left\langle\boldsymbol{r}^{2}\right\rangle=\frac{1}{12} n b^{2}=6 D^{\prime} n
$$

and

$$
D^{\prime}=\frac{b^{2}}{72}
$$

Consequently the distribution function $W(\boldsymbol{r}, n)$ obeys the following "diffusion" equation:

$$
\begin{aligned}
\frac{\partial W}{\partial n} & =D^{\prime}{V_{r}^{2} W}^{2} \\
& =D^{\prime}\left\{\frac{\partial^{2} W}{\partial r^{2}}+\frac{2}{r} \frac{\partial W}{\partial r}\right\}
\end{aligned}
$$

Assume that the whole chain is included in a sphere of radius $R$ around the center of mass of the chain. Since we have shown in the previous section that the effect of adsorption of the chain by the boundary becomes small in evaluating the width of the chain for large $n$, we adopt for simplicity the boundary condition (complete absorption)

$$
W(\boldsymbol{r}, n)=0 \quad \text { at } \quad r=R
$$

The solution of eq 25 under the boundary condition (26) and the initial condition

is give by

$$
W(\boldsymbol{r}, 0: R)=\delta(\boldsymbol{r})
$$

$$
\begin{aligned}
W(\boldsymbol{r}, n: R)= & \frac{1}{2 r R^{2}} \sum_{l=1}^{\infty} l \\
& \times \exp \left[-\frac{1}{72}\left(\frac{l \pi}{R}\right)^{2} n b^{2}\right] \sin \left(\frac{l \pi}{R} r\right)
\end{aligned}
$$

On the other hand, putting $m=(j-n / 2)^{2}$, one verifies the following equation for the function (21):

$$
\frac{\partial W}{\partial m}=\frac{b^{2}}{6 n} \nabla_{s_{j}}^{2} W\left(s_{j}, n\right)
$$

at $m=0, s_{j}=s_{n / 2}=r$ and $W\left(s_{n / 2}, n\right)$ is equal to the function $W(\boldsymbol{r}, n)$ given by (28). Therefore, $W\left(s_{j}, n: R\right)$ is given by

$$
W\left(s_{j}, n: R\right)=\int_{0 \leqq r \leqq R} P\left(s_{j}, m: \boldsymbol{r}, 0\right) W(\boldsymbol{r}, n: R) \mathrm{d} \boldsymbol{r}
$$

where

$$
\begin{aligned}
P\left(s_{j}, m: r, 0\right)= & \frac{1}{2 \pi s_{j} r R} \sum_{l=1}^{\infty} \exp \left[-\frac{b^{2}}{6 n} m\left(\frac{l_{\pi}}{R}\right)^{2}\right] \\
& \times \sin \left(\frac{l \pi}{R} s_{j}\right) \sin \left(\frac{l \pi}{R} r\right)
\end{aligned}
$$

is the elementary solution of (29) under the boundary condition

$$
W\left(s_{j}, n\right)=0, \quad \text { at } \quad s_{j}=\left|s_{j}\right|=R
$$

Thus we obtain

$$
\begin{aligned}
W\left(s_{j}, n: R\right) & =\frac{1}{2 s_{j} R^{2}} \sum_{l=1}^{\infty} l \\
& \times \exp \left[-\frac{n b^{2}}{6}\left\{\left(\frac{j}{n}-\frac{1}{2}\right)^{2}+\frac{1}{12}\right\}\left(\frac{l \pi}{R}\right)^{2}\right] \\
& \times \sin \left(\frac{l \pi}{R} s_{j}\right)
\end{aligned}
$$


The Widths of a Polymer Chain

Table I. Dimensions of the random Coil Chain

\begin{tabular}{|c|c|c|c|c|c|}
\hline \multirow{2}{*}{$\begin{array}{l}\text { Dimensions of the } \\
\text { random coil } \\
\text { chain }\end{array}$} & \multirow{2}{*}{$\begin{array}{l}x \text {-component of } \\
\text { end-to-end } \\
\text { distance, } R_{x}\end{array}$} & \multicolumn{2}{|r|}{ Width, $L$} & \multirow{2}{*}{$\begin{array}{c}\text { Radius of the } \\
\text { circumscribing } \\
\text { sphere around one } \\
\text { end, } R_{\mathrm{WKK}}(p=1)\end{array}$} & \multirow{2}{*}{$\begin{array}{l}\text { Diameter of the } \\
\text { circumscribing sphere } \\
\text { around the center } \\
\text { of mass, } 2 R(p=1)\end{array}$} \\
\hline & & $p=1 / 2^{\mathrm{b}}$ & $p=1$ & & \\
\hline $\begin{array}{l}\text { First moment } \\
\qquad(\sqrt{n} b)\end{array}$ & $\sqrt{\frac{2}{3 \pi}}=0.46^{\mathrm{a}}$ & 0.90 & $2 \sqrt{\frac{2}{3 \pi}}=0.92$ & $\frac{1}{2} \sqrt{\frac{\pi^{3}}{6}}=1.14$ & $\frac{1}{3} \sqrt{\frac{\pi^{3}}{2}}=1.31$ \\
\hline $\begin{array}{l}\text { Root mean square } \\
\text { of second moment } \\
\qquad(\sqrt{ } n b)\end{array}$ & $\frac{1}{\sqrt{3}}=0.58$ & 0.94 & $\sqrt{\frac{4}{3} \ln 2}=0.96$ & $\sqrt{\frac{7}{6} \zeta(3)}=1.18$ & $2 \sqrt{\frac{7}{18} \zeta(3)}=1.37$ \\
\hline
\end{tabular}

a We mean $\left\langle\left|R_{x}\right|\right\rangle$ for the average.

b We consider here the case of $p=1 / 2, \lambda=b / 2$, and $n=1000$.

This function for $m=(n / 2)^{2}$, or in other words for either $j=0$ or $n$, when integrated with respect to $s_{j}$ over the sphere of radius $R$, is the probability that the part of the chain from $n / 2$ to $n$ or from 0 to $n / 2$ lies completely in the sphere of radius $R$. Therefore

$$
\begin{aligned}
w_{n}(R) & =\frac{1}{2}\left[\int W\left(s_{n}, n: R\right) \mathrm{d} s_{n}+\int W\left(s_{0}, n: R\right) \mathrm{d} s_{0}\right] \\
& =2 \sum_{l=1}^{\infty}(-1)^{l-1} \exp \left[-\frac{n b^{2}}{18}\left(\frac{l \pi}{R}\right)^{2}\right]
\end{aligned}
$$

is the probability that the whole chain is contained in a sphere of radius $R$ around the center of mass.

Consequently the probability density $F(R)$ that the whole chain is contained in the sphere of radius $R$ around the center of mass and just makes contact with the sphere can be obtained from $w_{n}(R)$ to be

$$
\begin{aligned}
F(R) & =\frac{\partial w_{n}}{\partial R} \\
& =\frac{2}{9} n b^{2} \sum_{l=1}^{\infty}(-1)^{l-1} \frac{(l \pi)^{2}}{R^{3}} \exp \left[-\frac{n b^{2}}{18}\left(\frac{l \pi}{R}\right)^{2}\right]
\end{aligned}
$$

We may call this radius $R$ the radius of the circumscribing sphere around the center of mass. Figure 3 shows the relationship between $\sqrt{n} b F(R)$ and $R / \sqrt{n} b$. The first and the second moments of $F(R)$ are obtained respectively (as shown in Appendix B),

$$
\begin{gathered}
\langle R\rangle=\frac{1}{6} \sqrt{\frac{\pi^{3}}{2}} \sqrt{n} b=0.66 \sqrt{n} b \\
\left\langle R^{2}\right\rangle=\frac{7}{18} \zeta(3) n b^{2}=0.47 n b^{2}
\end{gathered}
$$

where $\zeta(k)$ is the zeta function

$$
\zeta(k)=\sum_{n=1}^{\infty} \frac{1}{n^{k}}
$$

From Figure 3 the most probable value $R_{m}$, where $F(R)$ takes the maximum value, is found to be given approximately by

$$
R_{m}=0.58 \sqrt{n} b
$$

This value is smaller than the first moment (eq 36).

\section{DISCUSSION}

As mentioned in the preceding section, Hollingsworth considered the radius of the circumscribing sphere around one end of the chain. Weidmann et al. gave exactly the expression of this radius (denoted as $R_{\mathrm{WKK}}$ ) for $p=1$.

$$
\begin{gathered}
\left\langle R_{\mathrm{WKK}}\right\rangle=\frac{1}{2} \sqrt{\frac{\pi^{3}}{6}} \sqrt{n} b \\
\left\langle R_{\mathrm{WKK}}^{2}\right\rangle=\frac{7}{6} \zeta(3) n b^{2}
\end{gathered}
$$

which can also be obtained by the present method (see Appendix C). The values $\left\langle R_{\mathrm{WKK}}\right\rangle$ and $\left\langle R_{\mathrm{WKK}}^{2}\right\rangle^{1 / 2}$ are $\sqrt{3}$ times as large as the values $\langle R\rangle$ and $\left\langle R^{2}\right\rangle^{1 / 2}$, given respectively by eqs 36 and 37 . In Table I various dimensions of the chain are compared.

\section{APPENDIX A}

The first moment of $f_{p}(L)$ is defined as

$$
\langle L\rangle_{p}=\int_{0}^{\infty} L f_{p}(L) \mathrm{d} L
$$


K. Moro and N. SAItô

Substituting eq 16 into (A-1), we obtain

where

$$
\begin{aligned}
\langle L\rangle_{p} & =\int_{0}^{\infty}\left(L^{2} w_{\mathrm{p}}{ }^{\prime \prime}+2 L w_{\mathrm{p}}{ }^{\prime}\right) \mathrm{d} L \\
& =\left[L^{2} w_{\mathrm{p}}{ }^{\prime}(L)\right]_{L=0}^{L=\infty} \\
& =\lim _{L \rightarrow \infty} L^{2} w_{\mathrm{p}}{ }^{\prime}(L)
\end{aligned}
$$

$$
\begin{aligned}
w_{\mathrm{p}}{ }^{\prime}(L)= & 4 \sum_{l=1, \mathrm{odd}}^{\infty}\left[\frac{\sin \alpha_{l}}{L \alpha_{l}\left(\alpha_{l}+\sin \alpha_{l}\right)^{3}}\right. \\
& \times\left\{\alpha_{l}{ }^{2} \sin \alpha_{l}+\left(\cos \alpha_{l}-1\right)\left(\alpha_{l}+\sin \alpha_{l}\right)\right\} \\
& \left.+\frac{2 \alpha_{l}{ }^{2}\left(1-\cos \alpha_{l}\right)}{L^{3}\left(\alpha_{l}+\sin \alpha_{l}\right)^{2}} D n\right] \exp \left(-\frac{\alpha_{l}{ }^{2}}{L^{2}} D n\right)
\end{aligned}
$$

From eq. 9 we have

$$
\sin \alpha_{l}=\frac{2}{\frac{\alpha_{l}}{a L}+\frac{a L}{\alpha_{l}}}, \quad \cos \alpha_{l}=\frac{\frac{\alpha_{l}}{a L}-\frac{a L}{\alpha_{l}}}{\frac{\alpha_{l}}{a L}+\frac{a L}{\alpha_{l}}}
$$

These are expanded in powers of $\beta_{l}=\alpha_{l} / a L$, as follows:

$$
\left.\begin{array}{l}
\sin \alpha_{l}=2 \beta_{l}\left(1-\beta_{l}{ }^{2}+\beta_{l}{ }^{4}+\cdots\right) \\
\cos \alpha_{l}=-1+2 \beta_{l}{ }^{2}-2 \beta_{l}{ }^{4}+\cdots
\end{array}\right\}
$$

Substituting (A-5) into (A-2), we have

$$
\begin{aligned}
\langle L\rangle_{p}= & \lim _{L \rightarrow \infty} \sum_{l=1, \text { odd }}^{\infty}\left[-\frac{16}{a(a L+2)^{2}}\left\{\frac{1}{\beta_{l}{ }^{2}}-a L\right.\right. \\
& \left.+\left(2 a L-7+\frac{20}{a L+2}-\frac{12}{(a L+2)^{2}}\right) \beta_{l}{ }^{2}+\cdots\right\} \\
& +\frac{16 a^{2} L D n}{(a L+2)^{2}}\left\{1-\left(1-\frac{4}{a L+2}\right) \beta_{l}{ }^{2}\right. \\
& \left.\left.+\left(1-\frac{8}{a L+2}+\frac{12}{(a L+2)^{2}}\right) \beta_{l}{ }^{4}+\cdots\right\}\right] \\
& \times \exp \left(-\beta_{l}{ }^{2} a^{2} D n\right)
\end{aligned}
$$

Expansion (A-5) holds for $\beta_{l}<1$, but by virtue of the presence of the factor $\exp \left(-\beta_{l}{ }^{2} a^{2} D n\right)$, expansion (A-6) is valid over all the values of $\beta_{l}$. In the limit of large $L$, we have

$$
\alpha_{l}=(2 l+1) \pi \quad \text { and } \quad \beta_{l}=\frac{(2 l+1) \pi}{a L}
$$

These enable us to evaluate the summations appearing in (A-6) as follows:

$\sum_{l=0}^{\infty} \exp \left(-\beta_{l}{ }^{2} a^{2} D n\right)$

$$
\begin{aligned}
& \simeq \frac{1}{2} \sum_{l=-\infty}^{\infty} \exp \left[-\frac{(2 l+1)^{2}}{L^{2}} D n \pi^{2}\right] \\
& =\frac{1}{2} \sum_{l=-\infty}^{\infty} \int_{-\infty}^{\infty} \exp \left[-\frac{D n \pi^{2}}{L^{2}}(2 x+1)^{2}\right] \cdot \delta(x-l) \mathrm{d} x \\
& =\frac{1}{4 \sqrt{D n \pi}}\left[1+2 \sum_{l=1}^{\infty}(-1)^{l} \exp \left(-\frac{L^{2}}{4 D n} l^{2}\right)\right]
\end{aligned}
$$

where use is made of the identity

$$
\sum_{l=-\infty}^{\infty} \delta(x-l)=1+2 \sum_{l=1}^{\infty} \cos 2 \pi l x
$$

in the integration of the second line. In the same way we have, for large $L$,

$$
\begin{aligned}
& \sum_{l=0}^{\infty} \beta_{l}{ }^{2} \exp \left[-\beta_{l}{ }^{2} a^{2} D n\right] \\
& \simeq \frac{1}{2} \sum_{l=-\infty}^{\infty} \frac{(2 l+1)^{2}}{a^{2} L^{2}} \pi^{2} \exp \left[-\frac{(2 l+1)^{2}}{L^{2}} D n \pi^{2}\right] \\
&= \frac{L}{8 a^{2} \sqrt{\pi}(\sqrt{D n})^{3}}\left[1+2 \sum_{l=1}^{\infty}(-1)^{l}\left(1-\frac{L^{2}}{2 D n} l^{2}\right)\right. \\
&\left.\times \exp \left(-\frac{L^{2}}{4 D n} l^{2}\right)\right]
\end{aligned}
$$

$\sum_{l=0}^{\infty} \beta_{l}{ }^{4} \exp \left[-\beta_{l}{ }^{2} a^{2} D n\right]$

$$
\begin{aligned}
\simeq & \frac{1}{2} \sum_{l=-\infty}^{\infty} \frac{(2 l+1)^{4}}{a^{4} L^{4}} \pi^{4} \exp \left[-\frac{(2 l+1)^{2}}{L^{2}} D n \pi^{2}\right] \\
= & \frac{3 L}{16 a^{4} \sqrt{\pi}(\sqrt{\bar{D}})^{5}}\left[1+2 \sum_{l=1}^{\infty}(-1)^{l}\left\{\frac{1}{12}\left(\frac{L^{2}}{D n} l^{2}\right)^{2}\right.\right. \\
& \left.\left.-\frac{L^{2}}{D n} l^{2}+1\right\} \exp \left(-\frac{L^{2}}{4 D n} l^{2}\right)\right]
\end{aligned}
$$

Further we have

$$
\begin{aligned}
\lim _{L \rightarrow \infty} & \frac{-16}{a(a L+2)^{2}} \sum_{l=0}^{\infty} \frac{1}{\beta_{l}{ }^{2}} \exp \left(-\beta_{l}{ }^{2} a^{2} D n\right) \\
= & \lim _{L \rightarrow \infty} \frac{-16}{a(a L+2)^{2}} \sum_{l=0}^{\infty} \frac{a^{2} L^{2}}{(2 l+1)^{2} \pi^{2}} \\
& \times \exp \left[-\frac{(2 l+1)^{2}}{L^{2}} \pi^{2} D n\right] \\
= & \lim _{L \rightarrow \infty}-\frac{16}{a \pi^{2}} \sum_{l=0}^{\infty} \frac{1}{(2 l+1)^{2}}=-\frac{2}{a}
\end{aligned}
$$

Substituting (A-8) and (A-10)-(A12) into (A-6), we obtain

$$
\begin{aligned}
\langle L\rangle_{p}= & 2 \sqrt{\frac{2}{3 \pi}} \sqrt{n} b\left[1-\frac{1}{2} \sqrt{\frac{\pi}{6}}\left(\frac{1-p}{p} \frac{b}{\lambda \sqrt{n}}\right)\right. \\
& +\frac{1}{12}\left(\frac{1-p}{p} \frac{b}{\lambda \sqrt{n}}\right)^{2} \\
& \left.-\frac{1}{144}\left(\frac{1-p}{p} \frac{b}{\lambda \sqrt{n}}\right)^{4}+\cdots\right]
\end{aligned}
$$


The Widths of a Polymer Chain

The second moment of $f_{p}(L)$ is also defined as

$$
\begin{aligned}
\left\langle L^{2}\right\rangle_{p} & =\int_{0}^{\infty} L^{2} f_{p}(L) \mathrm{d} L \\
& =\left[L^{3} w_{\mathrm{p}}{ }^{\prime}(L)\right]_{L=0}^{L=\infty}-\int_{0}^{\infty} L^{2} w_{\mathrm{p}}{ }^{\prime}(L) \mathrm{d} L
\end{aligned}
$$

The first term of (A-14) can be calculated in an expansion like (A-6), but the second term cannot, because $\alpha_{l}$ is now a function of the variable $L$. Numerical integration yields the valus

$$
\left\langle L^{2}\right\rangle_{1 / 2}=0.8864 n b^{2}
$$

for $n=1000$ and $p=1 / 2$. For $p=1$, however, we can proceed further. The function $w_{1}{ }^{\prime}$ is given from (A-3)

$$
w_{1}^{\prime}=\frac{16 D n}{L^{3}} \sum_{l=0}^{\infty} \exp \left[-\frac{D n \pi^{2}}{L^{2}}(2 l+1)^{2}\right]
$$

Then then first term of (A-14) is rewritten as

$$
\begin{aligned}
\lim _{L \rightarrow \infty} L^{3} w_{1}{ }^{\prime}(L) & =\lim _{L \rightarrow \infty} 16 D n L \int_{0}^{\infty} \exp \left[-4 D n \pi^{2} x^{2}\right] \mathrm{d} x \\
& =\lim _{L \rightarrow \infty} 4 \sqrt{\frac{D n}{\pi}} L
\end{aligned}
$$

The identity (A-9) enables us to rewrite the summation of $w_{1}(p)$ and calculate the integral of (A-14) as follows:

$$
\begin{aligned}
L^{2} w_{1}{ }^{\prime}(L)= & \frac{8 D n}{L} \int_{-\infty}^{\infty} \exp \left[-\frac{D n \pi^{2}}{L^{2}}(2 x+1)^{2}\right] \\
& \times \sum_{k=-\infty}^{\infty} \delta(x-k) \mathrm{d} x \\
= & 4 \sqrt{\frac{D n}{\pi}}+8 \sqrt{\frac{D n}{\pi}} \sum_{k=1}^{\infty}(-1)^{k} \\
& \times \exp \left[\frac{k^{2}}{4 D n} L^{2}\right]
\end{aligned}
$$

and

$$
\int_{0}^{\infty} L^{2} w_{1}^{\prime}(L) \mathrm{d} L=\lim _{L \rightarrow \infty} 4 \sqrt{\frac{D n}{\pi}} L+8 D n \sum_{k=1}^{\infty}(-1)^{k} \frac{1}{k}
$$

Although the first term on the rhs of (A-17) diverges as $L \rightarrow \infty$, it cancels with the first term of (A-14), i.e. (A-16). Thus we finally obtain

$$
\begin{aligned}
\left\langle L^{2}\right\rangle_{1} & =-8 D n \sum_{k=1}^{\infty}(-1)^{k} \frac{1}{k}=\frac{4}{3} \ln 2 n b_{2} \\
& \simeq 0.9242 n b^{2}
\end{aligned}
$$

\section{APPENDIX B}

$w_{n}(R)$ (eq 34) is rewritten as

$w_{n}(R)=-2 \sum_{l=1}^{\infty} \exp \left[-\frac{b n^{2}}{18}\left(\frac{l \pi}{R}\right)^{2}\right] \cos l \pi$

Then we obtain the following relation:

$$
\begin{aligned}
& 1-w_{n}(R)=\sum_{l=-\infty}^{\infty} \exp \left[-\frac{n b^{2}}{18}\left(\frac{l \pi}{R}\right)^{2}\right] \cos l \pi \\
& =\sum_{l=-\infty}^{\infty} \int_{-\infty}^{\infty} \delta(x-l) \exp \left[-\frac{n b^{2}}{18}\left(\frac{\pi}{R}\right)^{2} x^{2}\right] \cos \pi x \mathrm{~d} x \\
& =6 \sqrt{\frac{2 R^{2}}{\pi n b^{2}}} \sum_{l=1}^{\infty} \exp \left[-\frac{9}{2 n b^{2}}(2 l-1)^{2} R^{2}\right]
\end{aligned}
$$

where use is made of the identity (A-9). Since $w_{n}(R) \rightarrow 1$ for $R \rightarrow \infty$, the normalization of the function $F(R)$ is guaranteed. The first moment is calculated to be

$$
\begin{aligned}
\langle R\rangle & =\int_{0}^{\infty} R F(R) \mathrm{d} R=\int_{0}^{\infty} R \frac{\partial w_{n}}{\partial R} \mathrm{~d} R \\
& =-\int_{0}^{\infty} R \frac{\mathrm{d}}{\mathrm{d} R}\left[1-w_{n}(R)\right] \mathrm{d} R \\
& =\int_{0}^{\infty}\left[1-w_{n}(R)\right] \mathrm{d} R \\
& =\frac{2}{3} \sqrt{\frac{2 n b^{2}}{\pi}} \sum_{l=1}^{\infty} \frac{1}{(2 l-1)^{2}}=\frac{1}{6} \sqrt{\frac{\pi^{3}}{2}} \sqrt{n} b
\end{aligned}
$$

The last line follows from (B-2). Similarly the second moment is given by

$$
\begin{aligned}
\left\langle R^{2}\right\rangle & =\int_{0}^{\infty} R^{2} F(R) \mathrm{d} R \\
& =2 \int_{0}^{\infty} R\left[1-w_{n}(R)\right] \mathrm{d} R \\
& =\frac{4}{9} n b^{2} \sum_{l=1}^{\infty} \frac{1}{(2 l-1)^{3}} \\
& =\frac{7}{18} \zeta(3) n b^{2}=0.47 n b^{2}
\end{aligned}
$$

since $\sum_{l=1}^{\infty} 1 /(2 l-1)^{k}=\left(1-1 / 2^{k}\right) \zeta(k)$, where $\zeta(k)=$ $\sum_{l=1}^{\infty} 1 / l^{k}$ is the zeta function.

\section{APPENDIX C}

The radius $R_{\mathrm{WKK}}$ of the contact sphere around one terminal of the chain can be obtained when we consider eq 25 with $D^{\prime}=b^{2} / 6$ under 
the boundary condition (26) and the initial condition

$$
W(\boldsymbol{r}, 0)=\delta(\boldsymbol{r})
$$

By a similar procedure, the probability $w_{n}(R)_{\mathrm{WKK}}$ that the whole chain is contained in a sphere of radius $R_{\mathrm{WKK}}$ round one terminal of the chain is given by

$$
\begin{aligned}
& 1-w_{n}\left(R_{\mathrm{WKK}}\right) \\
& =1-2 \sum_{l=1}^{\infty}(-1)^{l+1} \exp \left[-\frac{n b^{2}}{6}\left(\frac{l \pi}{R_{\mathrm{W} \mathrm{KK}}}\right)^{2}\right] \\
& =2 \sqrt{\frac{6 R_{\mathrm{WKK}}^{2}}{n b^{2} \pi}} \sum_{l=1}^{\infty} \exp \left[-\frac{3}{2 n b^{2}}(2 l-1)^{2} R_{\mathrm{WKK}}^{2}\right]
\end{aligned}
$$

and the first and the second moments are respectively

$$
\begin{gathered}
\left\langle R_{\mathrm{WKK}}\right\rangle=\frac{1}{2} \sqrt{\frac{\pi^{3}}{6}} \sqrt{n} b \\
\left\langle R_{\mathrm{WKK}}^{2}\right\rangle=\frac{7}{6} \zeta(3) n b^{2}
\end{gathered}
$$

\section{REFERENCES}

1. S. F. Edwards, Proc. Phys. Soc., 91, 513 (1967).

2. S. F. Edwards, J. Phys., A1 15 (1968).
3. N. Saitô and Yi-der Chen, J. Chem. Phys., 59, 3701 (1973). See especially the last section.

4. S. F. Edwards, Proc. Phys. Soc., 85, 613 (1965).

5. S. F. Edwards, in "Critical Phenomena", M. S. Green and J. V. Sengers, Ed., NBS Publication 273, 1966, p 225.

6. N. Saitô, K. Takahashi, and Y. Yunoki, $J$. Phys. Soc. Japan, 22, 219 (1967).

7. C. A. Hollingsworth, J. Chem. Phys., 16, 544 (1948).

8. J. J. Weidmann, H. Kuhn, and W. Kuhn, J. Chim. Phys., 50, 226 (1953). This paper was brought to our attention by Dr. Y. Tagami, at the meeting of the Polymer Division, Physical Society of Japan, at Kôriyama, Oct. 1975, where the present paper was read. We wish to thank Dr. Y. Tagami.

9. G. Kirchhoff, "Vorlesungen über die Theorie der Wärme", vierter Band, B. G. Teubner, Leipzig, 1894, p 30.

10. R. J. Rubin, J. Chem. Phys., 56, 5747 (1972).

11. E. A. Di Marzio and R. J. Rubin, ibid., 55, 4318 (1971).

12. A. Ishihara, J. Phys. Soc. Japan, 5, 201 (1950).

13. P. Debye and F. Bueche, J. Chem. Phys., 20, 1337 (1952).

14. H. Yamakawa, "Modern Theory of Polymer Solutions", Harper \& Row, New York, 1971, p 21. 